\title{
The Incentive Mechanism Coordination Model of the Military Enterprises-Led Supply Chain
}

\author{
Li Jianmin \\ The School of Management, Hubei Business College, Wuhan, China
}

Email address:

ljm184@163.com

To cite this article:

Li Jianmin. The Incentive Mechanism Coordination Model of the Military Enterprises-Led Supply Chain. American Journal of Management Science and Engineering. Vol. 3, No. 5, 2018, pp. 76-83. doi: 10.11648/j.ajmse.20180305.16

Received: November 1, 2018; Accepted: December 5, 2018; Published: December 11, 2018

\begin{abstract}
Because of the inconsistent objectives of decision-makers and asymmetric information, the efforts and enthusiasm of the military supply chain members will not only affect their own benefits, but also produce adverse selection and moral hazard problems, and even affect the supply chain stable operation. Therefore, it is necessary to establish a reasonable and effective coordination rules and procedures, namely the incentive mechanism, in order to achieve the goal of supply chain system to meet the needs of military users. To stimulate the motivation and enthusiasm of each the supply chain member, and the military supply chain overall revenue also achieve the maximization, The paper applies the incentive mechanism design \& game theory, designs a transfer payment incentive mechanism model between the supply chain channel members according to the order quantity size, tries to change the expected profit objective function of the incented object, makes them to choose the optimal effort level coincided with the military supply chain total expected revenue, establishes a long-term cooperative partnership each other based on mutual trust and information sharing, gives full play to their respective advantages, achieves the supply chain collaboration efficiency improved purpose, and ultimately realizes the goal to improve the military logistic support capability. This study method mainly refers to the use of certain means to regulate economic interests to stimulate and restrain the decision-making behavior of military enterprises and warehouses, enhance and mobilize their motivation and enthusiasm. The study conclusion has important reference value to improve the coordination level of army and local and the military logistic support effectiveness.
\end{abstract}

Keywords: The Enterprises-Ledmilitary Supply Chain, Incentive Mechanism, Coordination

\section{Introduction}

Though the revenue-sharing mechanism can make the military supply chain to achieve coordination, increase the military supply chain overall revenue to more close to the centralized decision-making system revenue, also achieve the members revenue Pareto improvement. [1-2] However, the military enterprises and the rear warehouse two members of the military supply chain each belong to two different systems and decision-making interests body of the internal forces and external forces, different responsibilities, the military enterprises or warehouse, always improves the military supply chain overall efficiency on the premise of realizing their own interests maximization, inevitably produce decision twist, speculation and unethical behavior because of the interest driving, which are inconsistent and rebellious with the revenue-sharing mechanism to the whole alliance members interests request, thus damage or reduce the military supply chain overall efficiency, influence the supply chain system coordination level, make the alliance members income distribution problem become more complex, also greatly reduce the military logistics support benefit. [3] And both the military enterprises and the warehouses benefit goal function are inconsistent in military supply chain, own information is inadequate and incomplete each other, the entity for its own interests rather than with other entities share some important information, do not want to sacrifice their own interests to obtain the supply chain overall best interests, information processing time becoming longer, information processing number becoming more and more, information distortion becoming larger, the adverse selection and moral problems has not been eradicated, such as "double marginal effect", "bullwhip effect", "drag, drop and rise", "lazy", "not as", "work not hard" and "positive initiative not high". The chain members effort level and enthusiasm not only affect their own income, but also spread to the supply 
chain steady operation. [4] Therefore, need to establish a reasonable and effective coordination rules and procedures between the members, that is the incentive mechanism, which achieve the supply chain system to meet the target of the military users needs.

The mechanism mainly referring to certain economic interests means regulation, incentive and restraint the military enterprises and warehouses decision-making behavior, enhance and arouse their motivation and enthusiasm, raise the army and place coordination level, each other form strategic alliance based on mutual trust and information sharing, or establish long-term cooperative partnership, give full play to their respective advantages, achieve the military enterprises and warehouse interests and the military supply chain overall efficiency increasing, and ultimately improve the army logistics support benefit target. [5]

\section{The Military Supply Chain Performance Analysis in Decentralized Decision Making}

According to the order quantity size, design the transfer payment incentive mechanism between the chain members, try to change the incentive object expected profit objective function, allow them to choose the optimal effort level coinciding with the military supply chain total expected revenue. [6]

\subsection{Model Assumptions and Parameter Description}

1. build a Stackel-berg game model composed of a military enterprise (denoted as $S$ ) and warehouse $i(i=1,2)$, the military enterprises as the principal, the warehouse as the agent. The military enterprises and warehouses have independent decision-making power, the military enterprises supply the same type products to two warehouses at $w_{i}$ wholesale prices in a operation cycle, $c$ is the military enterprises unit production cost, then warehouses supply the product to the military users by storing at $p_{i}$ price, as shown in Figure 1. This constitutes a two-stage military supply chain game process. [7]

The military enterprises as a leader, enjoys the decide right of the benefit, income sharing ratio and the wholesale price. As a follower, the warehouse has the right to decide the product order quantity.

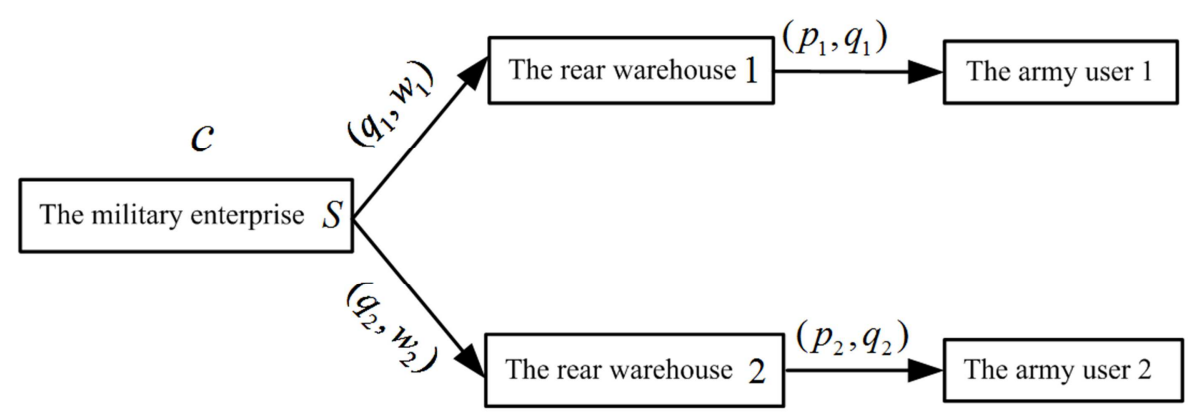

Figure 1. The two stage military supply chain game model.

2. The warehouse serves different army users, assuming that the order quantity $q_{i}\left(e_{i}\right)$ is related to the warehouse distribution service level (as following: referred to the effort level), the service user's fixed plan needs and random demand factors. Among, the random demand factors, such as the military combat training tasks temporary adjustment, the market and price changes, as well as the natural conditions changes, caused the demand etc., can be expressed by the formula $q_{i}\left(e_{i}\right)=A e_{i}+N_{i}+\lambda_{i}, \quad(i=1,2)$. Among them, one-dimensional variable $e_{i}$ represents the warehouse effort level, for warehouse the consuming weighted average value such as investment manpower, material resources and technology, specific performance in the improving its supplies support capability, service quality, to complete the logistics indicators; $A$ ( $A>0$ )on behalf of the warehouse effort level influence coefficient, namely the warehouse operation capacity coefficient; $N_{i}$ is a constant valve, which express the warehouse service user's fixed plan demand; $\lambda_{i}$ is a random variable, reflecting the uncertainty factors in the user demand process, are independent and have the same distribution density function $f\left(\lambda_{i}\right)$, for the simple reasons, supposed that $\lambda_{i}$ obeys uniform distribution on the interval $[l, h]$, namely $f\left(\lambda_{i}\right)=\frac{1}{h-l}$, and $E\left(\lambda_{i}\right)=0$.

3. TheWarehouse needs some input to improve its own distribution service level, convertible to effort cost to measure, used to $c\left(e_{i}\right)$ denotes the invested cost when the warehouse $i$ inputs the effort level $e_{i}$, let $c\left(e_{i}\right)=\frac{1}{2} b e_{i}^{2}, b$ is the effort cost coefficient, and $b>0, c(0)=0, c^{\prime}\left(e_{i}\right)>0$, $c^{\prime \prime}\left(e_{i}\right)>0$, which ensures that $c\left(e_{i}\right)$ is a monotone increasing convex function.

4. The military enterprises design the transfer payment incentive mechanism according to the warehouse order quantity size. $T$ indicates a reward for a warehouse that provides a large order amount, $t$ indicates a reward for a warehouse that provides a small order amount, and $T>t$. The purpose is to motivate the warehouse to choose the best effort level, to achieve the their revenue maximization. 
5. $\pi\left(q_{i}\left(e_{i}\right)\right), \pi_{s}\left(q_{i}\left(e_{i}\right)\right), \pi_{r}\left(q_{i}\left(e_{i}\right)\right)$ respectively is on behalf of the military supply chain, military enterprises, warehouses to obtain the desired revenue from the channel $i$. $\pi^{N}\left(q_{i}\left(e_{i}\right)\right), \pi_{s}^{N}\left(q_{i}\left(e_{i}\right)\right), \pi_{r}^{N}\left(q_{i}\left(e_{i}\right)\right)$ respectively is on behalf of the military supply chain, military enterprises, warehouses to obtain the maximum expected revenue from the channel $i$ in decentralized decision-making and no incentive mechanism, also known as the retained earnings. $\pi^{J}\left(q_{i}\left(e_{i}\right)\right), \pi_{s}^{J}\left(q_{i}\left(e_{i}\right)\right), \quad \pi_{r}^{J}\left(q_{i}\left(e_{i}\right)\right)$ respectively is on behalf of the military supply chain, military enterprises, warehouses to obtain the maximum expected revenue from the channel $i$ in centralized decision-making and no incentive mechanism. $\bar{\pi}\left(q_{i}\left(e_{i}\right)\right), \bar{\pi}_{s}\left(q_{i}\left(e_{i}\right)\right), \quad \bar{\pi}_{r}\left(q_{i}\left(e_{i}\right)\right)$ is respectively on behalf of the military supply chain, military enterprises, warehouses to obtain the maximum expected return from the channel $i$ in incentive mechanism. [8]

6. The military supply chain members are risk neutral, namely the utility level is equal to the expected return level.

7. $\left(\phi, \eta, w_{i}, b_{i}, q_{i}\right)$ is the revenue sharing mechanism that the military enterprises provides for the warehouse, of which $\eta$ is the distribution ratio that the warehouse $i$ income accounted for the military supply chain channel $i$ total revenue, and $\phi$ is the proportion that the warehouse $i$ income accounted for the channels $i$ total revenue. It is assumed that $\phi$ and $\eta$ is the proportion that the warehouse total revenue and income accounted for the military supply chain total income and total income, and the military enterprises proportion accounted for $1-\phi$ and $1-\eta \cdot[9]$

\subsection{Model Construction and Analysis}

The military supply chain members decision is usually not optimal in terms of the whole supply chain under the "double marginalization", and the supply chain overall efficiency needs to be improved.

By the above model assumptions and the relevant parameters definition, in the revenue sharing mechanism, the warehouse expected revenue function is:

$$
\begin{gathered}
\pi_{r}\left(q_{i}\left(e_{i}\right)\right)=E\left[\left(\eta p_{i} q_{i}\left(e_{i}\right)-w_{i} q_{i}\left(e_{i}\right)-\frac{1}{2} b e_{i}^{2}\right]\right. \\
=\left(\eta p_{i}-w_{i}\right)\left(A e_{i}+N_{i}\right)-\frac{1}{2} b e_{i}^{2}
\end{gathered}
$$

The military enterprises obtained desired revenue function from the channel $i$ can be expressed:

$$
\begin{gathered}
\pi_{s}\left(q_{i}\left(e_{i}\right)\right)=E\left\{\left[(1-\eta) p_{i}+w_{i}-c\right] q_{i}\left(e_{i}\right)\right\} \\
\left.=E\left\{\left[(1-\eta) p_{i}+w_{i}-c\right)\right]\left(A e_{i}+N_{i}+\lambda_{i}\right)\right\} \\
=\left[\left(p_{i}-c+w_{i}-\eta p_{i}\right)\left(A e_{i}+N_{i}\right)\right.
\end{gathered}
$$

The expected revenue function of the military supply chain channel $i$ is:

$$
\pi\left(q_{i}\left(e_{i}\right)\right)=\left(p_{i}-c\right)\left(A e_{i}+N_{i}\right)-\frac{1}{2} b e_{i}^{2}
$$

From the above we can know, $e_{i}$ is the decision variables, determines the effort level, and then determines the order quantity and the military supply chain channel $i$ efficiency.

The first derivative Formula (1), get

$$
\frac{d \pi_{r}\left(q_{i}\left(e_{i}\right)\right)}{d e_{i}}=A\left(\eta p_{i}-w_{i}\right)-b e_{i} .
$$

Formula (1) for the two order derivative, get $\frac{d^{2} \pi_{r}\left(q_{i}\left(e_{i}\right)\right)}{d e_{i}^{2}}=-b<0$.

So $\pi_{r}\left(q_{i}\left(e_{i}\right)\right)$ is a concave function, there is a maximum value point. Let $\frac{d \pi_{r}\left(q_{i}\left(e_{i}\right)\right)}{d e_{i}}=A\left(\eta p_{i}-w_{i}\right)-b e_{i}=0$, we can obtained the optimal effort level of decentralized decision-making without incentive mechanism, $e_{i}^{N}=\frac{A\left(\eta p_{i}-w_{i}\right)}{b}$. Under the revenue sharing mechanism, $e_{i}^{N}$ namely is the best effort level of the rational warehouse. Thus, the warehouse expected revenue function is:

$$
\pi_{r}^{N}\left(q_{i}\left(e_{i}\right)\right)=\frac{A^{2}\left(\eta p_{i}-w_{i}\right)^{2}}{2 b}+N_{i}\left(\eta p_{i}-w_{i}\right) .
$$

At this point, the military enterprises obtained desired revenue function from the channel $i$ is:

$$
\begin{aligned}
& \pi_{s}^{N}\left(q_{i}\left(e_{i}\right)\right)= \frac{A^{2}\left(\eta p_{i}-w_{i}\right)\left(p_{i}-c+w_{i}-\eta p_{i}\right)}{b}+ \\
& N_{i}\left(p_{i}-c+w_{i}-\eta p_{i}\right)
\end{aligned}
$$

The expected revenue function of the military supply chain channel $i$ is:

$$
\begin{gathered}
\pi^{N}\left(q_{i}\left(e_{i}\right)\right)=\frac{A^{2}\left(p_{i}-c\right)\left(\eta p_{i}-w_{i}\right)}{b}+N_{i}\left(p_{i}-c\right) \\
-\frac{A^{2}\left(\eta p_{i}-w_{i}\right)^{2}}{2 b} .
\end{gathered}
$$

Also because $\pi^{\prime}\left(q_{i}\left(e_{i}\right)\right)=A\left(p_{i}-c\right)-b e_{i} \quad, \quad$ and $\pi^{\prime \prime}\left(q_{i}\left(e_{i}\right)\right)=-b<0, \pi\left(q_{i}\left(e_{i}\right)\right)$ is a concave function, There is a great value point. Set to $e_{i}^{*}$, let $\pi^{\prime}\left(q_{i}\left(e_{i}\right)\right)=0$, get $e_{i}^{*}=\frac{A\left(p_{i}-c\right)}{b}$.

$e_{i}^{*}$ may be understood as the effort level of the military supply chain channel $i$ to obtain the maximum revenue 
under the centralized decision. At this point, $e_{i}^{*}>e_{i}^{N}$, namely $\frac{A\left(p_{i}-c\right)}{b}>\frac{A\left(\eta p_{i}-w_{i}\right)}{b}$, means that the warehouse efforts level under the decentralized decision-making is lower than the level of centralized decision-making, there is still room improvement. At the same time, can obtain $w_{i}>\eta p_{i}-p_{i}+c$.

When $e_{i}^{*}=\frac{A\left(p_{i}-c\right)}{b}$, the warehouse $i$ expected revenue is:

$$
\begin{gathered}
\pi_{r}\left(q_{i}\left(e_{i}\right)\right)_{e_{i}=e_{i}^{*}}=\frac{A^{2}\left(\eta p_{i}-w_{i}\right)\left(p_{i}-c\right)}{b}+N_{i}\left(\eta p_{i}-w_{i}\right)- \\
\frac{A^{2}\left(p_{i}-c\right)^{2}}{2 b}<\pi_{r}^{N}\left(q_{i}\left(e_{i}\right)\right) .
\end{gathered}
$$

The above analysis shows that there is no consensus on the warehouse effort level under the decentralized decision and centralized decision making when there is no incentive mechanism, and the military supply chain can not reach the coordination. In the information symmetry, even if the military supply chain does not take any incentive measures, the rational warehouse will not hesitate to choose the optimal effort level to maximize their own revenue, namely the effort level $e_{i}^{N}$ in decentralized decision-making. [10]

\section{The Military Supply Chain Performance Analysis Under Centralized Decision Making}

\subsection{Model Construction and Analysis}

Step1: solve the military supply chain channel $i$ optimal effort level situation under centralized decision condition.

$$
\pi\left(q_{i}\left(e_{i}\right)\right)=\max _{e_{i}}\left[\left(p_{i}-c\right)\left(A e_{i}+N_{i}\right)-\frac{1}{2} b e_{i}^{2}\right]
$$

Among it, because $\frac{\partial^{2} \pi\left(q_{i}\left(e_{i}\right)\right)}{\partial e_{i}^{2}}=-b<0$, indicates that $\pi\left(q_{i}\left(e_{i}\right)\right)$ is a concave function, and there is a maximum value point.

$$
\begin{aligned}
& \text { let } \frac{\partial \pi\left(q_{i}\left(e_{i}\right)\right)}{\partial e_{i}}=A\left(p_{i}-c\right)-b e_{i}=0 \text {, get, } \\
& e_{i}^{J}=\frac{A\left(p_{i}-c\right)}{b}
\end{aligned}
$$

At this time, the military supply chain channels $i$ expected revenue is:

$$
\pi^{J}\left(q_{i}\left(e_{i}\right)\right)=\frac{A^{2}\left(p_{i}-c\right)^{2}}{2 b}+N_{i}\left(p_{i}-c\right)
$$

The military enterprises $S$ obtained the expected revenue from the channel $i$ is:

$$
\begin{gathered}
\left.\pi_{s}^{J}\left(q_{i}\left(e_{i}\right)\right)=\left[(1-\eta) p_{i}+w_{i}-c\right)\right] \frac{A^{2}\left(p_{i}-c\right)}{b}+ \\
\left.N_{i}\left[(1-\eta) p_{i}+w_{i}-c\right)\right]
\end{gathered}
$$

The warehouse $i$ expected revenue is:

$$
\begin{gathered}
\pi_{r}^{J}\left(q_{i}\left(e_{i}\right)\right)=\frac{A^{2}\left(p_{i}-c\right)\left(\eta p_{i}-w_{i}\right)}{b}+ \\
N_{i}\left(\eta p_{i}-w_{i}\right)-\frac{A^{2}\left(p_{i}-c\right)^{2}}{2 b}
\end{gathered}
$$

Step 2: according to the previous assumption $p_{i}>w_{i}>c$, get $p_{i}-c>p_{i}-w_{i}$, from the previous formula (5), $e_{i}^{J}=\frac{A\left(p_{i}-c\right)}{b}$, can obtain $e_{i}^{J}=\frac{A\left(p_{i}-c\right)}{b}>\frac{A\left(\eta p_{i}-w_{i}\right)}{b}$, and because of $e_{i}^{N}=\frac{A\left(\eta p_{i}-w_{i}\right)}{b}, e_{i}^{N}$ is the level effort under the incentive mechanism, at this time $e_{i}^{J} \neq e_{i}^{N}$, then continue to Step3.

Step 3: the military enterprises and warehouses determine the final revenue distribution ratio through negotiations. Assuming that the warehouse cooperative distribution proportion is $\phi_{j}(j=1,2), 0 \leq \phi_{j} \leq 1$, then the military enterprise cooperative revenue distribution ratio should be $1-\phi_{1}-\phi_{2}$. The revenue distribution proportion is related to the member position and negotiation ability in the military supply chain. [11-12]

Step 4: calculate the military supply chain revenue total increased quantity $\Delta U$.

$$
\begin{gathered}
\Delta U=\sum_{i=1}^{2}\left[\pi^{J}\left(q_{i}\left(e_{i}\right)\right)-\pi^{N}\left(q_{i}\left(e_{i}\right)\right)\right] \\
=\sum_{i=1}^{2} \frac{A^{2}\left[\left(p_{i}-c\right)+\left(w_{i}-\eta p_{i}\right)\right]^{2}}{2 b}
\end{gathered}
$$

Then, the warehouse and military enterprises cooperation revenue respectively is:

$$
\begin{aligned}
& D_{1}=\phi_{1} \times \Delta U=\phi_{1} \sum_{i=1}^{2} \frac{A^{2}\left[\left(p_{i}-c\right)+\left(w_{i}-\eta p_{i}\right)\right]^{2}}{2 b}, \\
& D_{2}=\phi_{2} \times \Delta U=\phi_{2} \sum_{i=1}^{2} \frac{A^{2}\left[\left(p_{i}-c\right)+\left(w_{i}-\eta p_{i}\right)\right]^{2}}{2 b}
\end{aligned}
$$




$$
\begin{gathered}
D_{3}=\left(1-\phi_{1}-\phi_{2}\right) \times \Delta U \\
=\left(1-\phi_{1}-\phi_{2}\right) \sum_{i=1}^{2} \frac{A^{2}\left[\left(p_{i}-c\right)+\left(w_{i}-\eta p_{i}\right)\right]^{2}}{2 b} .
\end{gathered}
$$

Step5: the military enterprises designs the transfer payment incentive mechanism $T$ and $t$ according to the order quantity size. Then, calculate the average transfer payment $V_{1}$ and $V_{2}$ for each warehouse:

$$
\begin{aligned}
& V_{1}=T \times \operatorname{prob}\left(q_{1}\left(e_{1}\right)>q_{2}\left(e_{2}\right)\right)+t \times \operatorname{prob}\left(q_{1}\left(e_{1}\right)<q_{2}\left(e_{2}\right)\right), \\
& V_{2}=T \times \operatorname{prob}\left(q_{2}\left(e_{2}\right)>q_{1}\left(e_{1}\right)\right)+t \times \operatorname{prob}\left(q_{2}\left(e_{2}\right)<q_{1}\left(e_{1}\right)\right) .
\end{aligned}
$$

Among them, $\operatorname{prob}\left(q_{1}\left(e_{1}\right)>q_{2}\left(e_{2}\right)\right)$ is the probability when $q_{1}\left(e_{1}\right)>q_{2}\left(e_{2}\right)$.

Step6: the each member expected payoff function under the transfer payment incentive mechanism is $\max E\left[D_{i}+T\left(q_{i}\left(e_{i}\right)\right)\right]$.

Step7:Verify the transfer payment incentive mechanism effectiveness. Mainly through whether it can realize the reasonable income division, whether it can really play the effective incentives role to judge. Namely, $\pi_{r}^{J}\left(q_{1}\left(e_{1}\right)\right)-\pi_{r}^{N}\left(q_{1}\left(e_{1}\right)\right)+V_{1} \geq D_{1}, \pi_{r}^{J}\left(q_{2}\left(e_{2}\right)\right)-\pi_{r}^{N}\left(q_{2}\left(e_{2}\right)\right)+V_{2} \geq D_{2}$, can get when respectively makes an equality:

$$
\begin{gathered}
V_{1}=D_{1}+\pi_{r}^{N}\left(q_{1}\left(e_{1}\right)\right)-\pi_{r}^{J}\left(q_{1}\left(e_{1}\right)\right) \\
=\phi_{1} \sum_{i=1}^{2} \frac{A^{2}\left[\left(p_{i}-c\right)+\left(w_{i}-\eta p_{i}\right)\right]^{2}}{2 b} \\
+\frac{A^{2}\left[\left(p_{1}-c\right)+\left(w_{1}-\eta p_{1}\right)\right]^{2}}{2 b}
\end{gathered}
$$

Also because,

$$
\begin{gathered}
V_{1}=T \times \operatorname{prob}\left(q_{1}\left(e_{1}\right)>q_{2}\left(e_{2}\right)\right)+t \times \operatorname{prob}\left(q_{1}\left(e_{1}\right)<q_{2}\left(e_{2}\right)\right) \\
=T \times \operatorname{prob}\left(q_{1}\left(e_{1}\right)>q_{2}\left(e_{2}\right)\right)+t \times\left[1-\operatorname{prob}\left(q_{1}\left(e_{1}\right)>q_{2}\left(e_{2}\right)\right)\right] \\
=(T-t) \times \operatorname{prob}\left(q_{1}\left(e_{1}\right)>q_{2}\left(e_{2}\right)\right)+t
\end{gathered}
$$

Therefore,

$$
\begin{aligned}
& (T-t) \times \operatorname{prob}\left(q_{1}\left(e_{1}\right)>q_{2}\left(e_{2}\right)\right)+t \\
= & \phi_{1} \sum_{i=1}^{2} \frac{A^{2}\left[\left(p_{i}-c\right)+\left(w_{i}-\eta p_{i}\right)\right]^{2}}{2 b}+\frac{A^{2}\left[\left(p_{1}-c\right)+\left(w_{1}-\eta p_{1}\right)\right]^{2}}{2 b}
\end{aligned}
$$

Similarly,

$$
\begin{gathered}
V_{2}=D_{2}+\pi_{r}^{N}\left(q_{2}\left(e_{2}\right)\right)-\pi_{r}^{J}\left(q_{2}\left(e_{2}\right)\right) \\
=\phi_{2} \sum_{i=1}^{2} \frac{A^{2}\left[\left(p_{i}-c\right)+\left(w_{i}-\eta p_{i}\right)\right]^{2}}{2 b}+ \\
\frac{A^{2}\left[\left(p_{2}-c\right)+\left(w_{2}-\eta p_{2}\right)\right]^{2}}{2 b}
\end{gathered}
$$

Then,

$$
\begin{gathered}
(T-t) \times \operatorname{prob}\left(q_{2}\left(e_{2}\right)>q_{1}\left(e_{1}\right)\right)+t \\
=\phi_{2} \sum_{i=1}^{2} \frac{A^{2}\left[\left(p_{i}-c\right)+\left(w_{i}-\eta p_{i}\right)\right]^{2}}{2 b}+ \\
\frac{A^{2}\left[\left(p_{2}-c\right)+\left(w_{2}-\eta p_{2}\right)\right]^{2}}{2 b}
\end{gathered}
$$

equations (8) plus equations (9):

$$
T+t=\left(\phi_{1}+\phi_{2}+1\right) \sum_{i=1}^{2} \frac{A^{2}\left[\left(p_{i}-c\right)+\left(w_{i}-\eta p_{i}\right)\right]^{2}}{2 b}
$$

$$
\begin{gathered}
\text { Also because }\left[\frac{\partial \pi_{r}\left(q_{i}\left(e_{i}\right)\right)}{\partial e_{i}}\right]_{e_{i}=e^{J}}=A\left(\eta p_{i}-w_{i}-p_{i}+c\right), \\
\left(\frac{\partial V_{i}}{\partial e_{i}}\right)_{e_{i}=e^{J}}=\frac{T-t}{h-l} A .
\end{gathered}
$$

Also let $\left(\frac{\partial \pi_{r}\left(q_{i}\left(e_{i}\right)\right)}{\partial e_{i}}\right)_{e_{i}=e^{J}}+\left(\frac{\partial V_{i}}{\partial e_{i}}\right)_{e_{i}=e^{J}}=0$, may get,

$$
\begin{gathered}
T+t=\left(\phi_{1}+\phi_{2}+1\right) \sum_{i=1}^{2} \frac{A^{2}\left[\left(p_{i}-c\right)+\left(w_{i}-\eta p_{i}\right)\right]^{2}}{2 b} \\
T-t=(h-l)\left[\left(p_{i}-c\right)+\left(w_{i}-\eta p_{i}\right)\right]
\end{gathered}
$$

Solve the equations composed of formula (11) and (12), get:

$$
\begin{gathered}
T=\left(\phi_{1}+\phi_{2}+1\right) \sum_{i=1}^{2} \frac{A^{2}\left[\left(p_{i}-c\right)+\left(w_{i}-\eta p_{i}\right)\right]^{2}}{4 b}+ \\
\frac{(h-l)\left[\left(p_{i}-c\right)+\left(w_{i}-\eta p_{i}\right)\right]}{2} \\
t=\left(\phi_{1}+\phi_{2}+1\right) \sum_{i=1}^{2} \frac{A^{2}\left[\left(p_{i}-c\right)+\left(w_{i}-\eta p_{i}\right)\right]^{2}}{4 b}- \\
\frac{(h-l)\left[\left(p_{i}-c\right)+\left(w_{i}-\eta p_{i}\right)\right]}{2}
\end{gathered}
$$

Under the transfer payment incentive mechanism, the military enterprises expected revenue is:

$$
\begin{aligned}
& \bar{\pi}_{s}\left(q_{1}\left(e_{1}\right), q_{2}\left(e_{2}\right)\right)=\pi_{s}^{J}\left(q_{1}\left(e_{1}\right)\right)+\pi_{s}^{J}\left(q_{2}\left(e_{2}\right)\right)-(T+t) \\
= & \sum_{i=1}^{2}\left\{\frac{A^{2}\left(p_{i}-c\right)\left[\left(p_{i}-c\right)+\left(w_{i}-\eta p_{i}\right)\right]}{b}+N_{i}\left[\left[\left(p_{i}-c\right)\right.\right.\right. \\
+ & \left.\left.\left(w_{i}-\eta p_{i}\right)\right]\right\}-\left(\phi_{1}+\phi_{2}+1\right) \sum_{i=1}^{2} \frac{A^{2}\left[\left(p_{i}-c\right)+\left(w_{i}-\eta p_{i}\right)\right]^{2}}{2 b}
\end{aligned}
$$


The NO.1 warehouse expected revenue is:

$$
\begin{gathered}
\bar{\pi}_{r}\left(q_{1}\left(e_{1}\right)\right)=\pi_{r}^{J}\left(q_{1}\left(e_{1}\right)\right)+\frac{T+t}{2} \\
=\left[\frac{A^{2}\left(p_{1}-c\right)\left(\eta p_{1}-w_{1}\right)}{b}+N_{1}\left(\eta p_{1}-w_{1}\right)-\frac{A^{2}\left(p_{1}-c\right)^{2}}{2 b}\right] \\
+\left(\phi_{1}+\phi_{2}+1\right) \sum_{i=1}^{2} \frac{A^{2}\left[\left(p_{i}-c\right)+\left(w_{i}-\eta p_{i}\right)\right]^{2}}{4 b}
\end{gathered}
$$

The NO.2 warehouse expected revenue is:

$$
\begin{gathered}
\bar{\pi}_{r}\left(q_{2}\left(e_{2}\right)\right)=\pi_{r}^{J}\left(q_{2}\left(e_{2}\right)\right)+\frac{T+t}{2} \\
=\left[\frac{A^{2}\left(p_{2}-c\right)\left(\eta p_{2}-w_{2}\right)}{b}+N_{2}\left(\eta p_{2}-w_{2}\right)-\frac{A^{2}\left(p_{2}-c\right)^{2}}{2 b}\right] \\
+\left(\phi_{1}+\phi_{2}+1\right) \sum_{i=1}^{2} \frac{A^{2}\left[\left(p_{i}-c\right)+\left(w_{i}-\eta p_{i}\right)\right]^{2}}{4 b}
\end{gathered}
$$

And because, the military enterprises total expected revenue under no incentive mechanism is:

$$
\begin{gathered}
\pi_{s}^{N}\left(q_{1}\left(e_{1}\right), q_{2}\left(e_{2}\right)\right)=\pi_{s}^{N}\left(q_{1}\left(e_{1}\right)\right)+\pi_{s}^{N}\left(q_{1}\left(e_{1}\right)\right) \\
=\sum_{i=1}^{2}\left[\frac{A^{2}\left(\eta p_{i}-w_{i}\right)\left(p_{i}-c+w_{i}-\eta p_{i}\right)}{b}+N_{i}\left(p_{i}-c+w_{i}-\eta p_{i}\right)\right]
\end{gathered}
$$

So,

$$
\begin{gathered}
\bar{\pi}_{s}\left(q_{1}\left(e_{1}\right), q_{2}\left(e_{2}\right)\right)-\pi_{s}^{N}\left(q_{1}\left(e_{1}\right), q_{2}\left(e_{2}\right)\right) \\
=\left(1-\phi_{1}-\phi_{2}\right) \sum_{i=1}^{2} \frac{A^{2}\left[\left(p_{i}-c\right)+\left(w_{i}-\eta p_{i}\right)\right]^{2}}{2 b} \geq 0 .
\end{gathered}
$$

Namely, $\quad \bar{\pi}_{s}\left(q_{1}\left(e_{1}\right), q_{2}\left(e_{2}\right)\right)>\pi_{s}^{N}\left(q_{1}\left(e_{1}\right), q_{2}\left(e_{2}\right)\right), \quad$ the military enterprises expected revenue under the incentive mechanism is greater than the level of the incentive mechanism.

And because, the expected revenue sum of the two warehouses under the no incentive mechanism is:

$$
\begin{gathered}
\pi_{r}^{N}\left(q_{1}\left(e_{1}\right), q_{2}\left(e_{2}\right)\right)=\pi_{r}^{N}\left(q_{1}\left(e_{1}\right)\right)+\pi_{r}^{N}\left(q_{2}\left(e_{2}\right)\right) \\
=\sum_{i=1}^{2}\left[\frac{A^{2}\left(\eta p_{i}-w_{i}\right)^{2}}{2 b}+N_{i}\left(\eta p_{i}-w_{i}\right)\right] \text { Then, } \\
\bar{\pi}_{r}\left(q_{1}\left(e_{1}\right), q_{2}\left(e_{2}\right)\right)-\pi_{r}^{N}\left(q_{1}\left(e_{1}\right), q_{2}\left(e_{2}\right)\right) \\
=\left[\bar{\pi}_{r}\left(q_{1}\left(e_{1}\right)\right)-\pi_{r}^{N}\left(q_{1}\left(e_{1}\right)\right)\right]+\left[\bar{\pi}_{r}\left(q_{2}\left(e_{2}\right)\right)-\pi_{r}^{N}\left(q_{2}\left(e_{2}\right)\right)\right] \\
=\left(\phi_{1}+\phi_{2}\right) \sum_{i=1}^{2} \frac{A^{2}\left[\left(p_{i}-c\right)+\left(w_{i}-\eta p_{i}\right)\right]^{2}}{2 b} \geq 0 .
\end{gathered}
$$

Namely, $\quad \bar{\pi}_{r}\left(q_{1}\left(e_{1}\right), q_{2}\left(e_{2}\right)\right) \geq \pi_{r}^{N}\left(q_{1}\left(e_{1}\right), q_{2}\left(e_{2}\right)\right), \quad$ the warehouse expected revenue under the incentive mechanism is greater than the level of the no incentive mechanism.

And because, the military supply chain system expected revenue under the no incentive mechanism is:

$$
\begin{gathered}
\pi^{N}\left(q_{1}\left(e_{1}\right), q_{2}\left(e_{2}\right)\right)=\pi^{N}\left(q_{1}\left(e_{1}\right)\right)+\pi^{N}\left(q_{2}\left(e_{2}\right)\right) \\
=\sum_{i=1}^{2}\left[\frac{A\left(p_{i}-c\right)\left(\eta p_{i}-w_{i}\right)}{b}+N_{i}\left(p_{i}-c\right)-\frac{A^{2}\left(\eta p_{i}-w_{i}\right)^{2}}{2 b}\right]
\end{gathered}
$$

The military supply chain expected revenue under the incentive mechanism is:

$$
\begin{gathered}
\bar{\pi}\left(q_{1}\left(e_{1}\right), q_{2}\left(e_{2}\right)\right)=\bar{\pi}\left(q_{1}\left(e_{1}\right)\right)+\bar{\pi}\left(q_{2}\left(e_{2}\right)\right) \\
=\sum_{i=1}^{n}\left[\frac{A^{2}\left(p_{i}-c\right)^{2}}{2 b}+N_{i}\left(p_{i}-c\right)\right]
\end{gathered}
$$

Then,

$$
\begin{aligned}
& \bar{\pi}\left(q_{1}\left(e_{1}\right), q_{2}\left(e_{2}\right)\right)-\pi^{N}\left(q_{1}\left(e_{1}\right), q_{2}\left(e_{2}\right)\right) \\
& =\sum_{i=1}^{2} \frac{A^{2}\left[\left(p_{i}-c\right)+\left(w_{i}-\eta p_{i}\right)\right]^{2}}{2 b} \geq 0,
\end{aligned}
$$

Namely, $\bar{\pi}\left(q_{1}\left(e_{1}\right), q_{2}\left(e_{2}\right)\right) \geq \pi^{N}\left(q_{1}\left(e_{1}\right), q_{2}\left(e_{2}\right)\right)$, the military supply chain expected revenue under the incentive mechanism is better than that of the no incentive mechanism.

At the same time, it can also proved that:

$$
\begin{gathered}
\bar{\pi}\left(q_{1}\left(e_{1}\right), q_{2}\left(e_{2}\right)\right)=\bar{\pi}_{s}\left(q_{1}\left(e_{1}\right), q_{2}\left(e_{2}\right)\right)+\bar{\pi}_{r}\left(q_{1}\left(e_{1}\right), q_{2}\left(e_{2}\right)\right), \\
\bar{\pi}_{r}\left(q_{1}\left(e_{1}\right), q_{2}\left(e_{2}\right)\right)=\left(\phi_{1}+\phi_{2}\right) \bar{\pi}\left(q_{1}\left(e_{1}\right), q_{2}\left(e_{2}\right)\right) .
\end{gathered}
$$

According to the above analysis, we can obtain that the military enterprises and warehouses in the transfer payment incentive mechanism and revenue sharing mechanism gain more than under decentralized decision and no incentive mechanism, indicating that the transfer payment incentive mechanism can make the supply chain coordination. Not only the military enterprises revenue achieves maximum, but also the warehouse income also achieves the optimal, and the military supply chain expected revenue achieves maximization. [13]

\subsection{Model Development}

Select $T\left(q_{i}\left(e_{i}\right)\right)=\alpha_{i}+\beta_{i}\left(p_{i}-c_{i}\right) q_{i}\left(e_{i}\right)$, Among them, $T\left(q_{i}\left(e_{i}\right)\right)$ is the total reward for the military enterprises provide to the warehouse $i$ (i.e. the warehouse total income), $\alpha_{i}$ is the fixed compensation (nothing to do with the output), $\beta_{i}$ is the motivate strength coefficient, represents that the warehouse enjoys the military supply chain final income ratio, namely that the military supply chain increase one unit of output, the warehouse will increase $\beta_{i}\left(p_{i}-c_{i}\right)$ 
income $^{[6]}$. So, the expectation of the linear transfer payment incentive mechanism is:

$$
\begin{gathered}
E\left[T\left(q_{i}\left(e_{i}\right)\right)\right]=E\left[\alpha_{i}+\beta_{i}\left(p_{i}-c\right)\left(A e_{i}+N_{i}+\lambda_{i}\right)\right] \\
=\alpha_{i}+\beta_{i}\left(p_{i}-c\right)\left(A e_{i}^{J}+N_{i}\right) .
\end{gathered}
$$

Select and design appropriate linear transfer payment incentive parameters $\alpha_{i}$ and $\beta_{i}$, make them satisfied the following formula,

$$
\begin{gathered}
E\left[T\left(q_{i}\left(e_{i}\right)\right)\right]=\alpha_{i}+\beta_{i}\left(p_{i}-c\right)\left(A e_{i}^{J}+N_{i}\right) \\
=D_{i}+\pi_{r}^{N}\left(q_{i}\left(e_{i}\right)\right)-\pi_{r}^{J}\left(q_{i}\left(e_{i}\right)\right) .
\end{gathered}
$$

According to the previous calculation process, it can be concluded that,

$$
\begin{gathered}
E\left[T\left(q_{i}\left(e_{i}\right)\right)\right]=\alpha_{i}+\beta_{i}\left(p_{i}-c\right)\left(A e_{i}^{J}+N_{i}\right) \\
=\phi_{j} \sum_{i=1}^{2} \frac{A^{2}\left[\left(p_{i}-c\right)+\left(w_{i}-\eta p_{i}\right)\right]^{2}}{2 b}+
\end{gathered}
$$

$$
\frac{A^{2}\left[\left(p_{i}-c\right)+\left(w_{i}-\eta p_{i}\right)\right]^{2}}{2 b}
$$

Because,

$$
\begin{aligned}
{\left[\frac{d \pi_{r}\left(q_{i}\left(e_{i}\right)\right)}{d e_{i}}\right]_{e_{i}=e_{i}^{J}} } & =A\left(\eta p_{i}-w_{i}-p_{i}+c\right) \\
\frac{d\left[T\left(q_{i}\left(e_{i}\right)\right)\right]}{d e_{i}} & =A \beta_{i}\left(p_{i}-c\right),
\end{aligned}
$$

then,

$$
\begin{gathered}
{\left[\frac{d \pi_{r}\left(q_{i}\left(e_{i}\right)\right)}{d e_{i}}\right]_{e_{i}=e_{i}^{J}}+\left[\frac{d\left[T\left(q_{i}\left(e_{i}\right)\right)\right]}{d e_{i}}\right]_{e_{i}=e_{i}^{J}}} \\
=\left(\eta p_{i}-w_{i}-p_{i}+c\right)+\beta_{i}\left(p_{i}-c\right)=0
\end{gathered}
$$

get $0<\beta_{i}=\frac{\left(p_{i}-c+w_{i}-\eta p_{i}\right)}{p_{i}-c}<1, \quad$ substitution it to formula (13), get,

$$
\alpha_{i}=\phi_{j} \sum_{i=1}^{2} \frac{A^{2}\left[\left(p_{i}-c\right)+\left(w_{i}-\eta p_{i}\right)\right]^{2}}{2 b}+\frac{A^{2}\left[\left(p_{i}-c\right)+\left(w_{i}-\eta p_{i}\right)\right]^{2}}{2 b}-\frac{A^{2}\left(p_{i}-c\right)\left[\left(p_{i}-c\right)+\left(w_{i}-\eta p_{i}\right)\right]}{b}-N_{i}\left[\left(p_{i}-c\right)+\left(w_{i}-\eta p_{i}\right)\right]
$$

So,

$$
\begin{gathered}
E\left[T\left(q_{i}\left(e_{i}\right)\right]=\alpha_{i}+\frac{A^{2}\left(p_{i}-c\right)\left(p_{i}-c+w_{i}-\eta p_{i}\right)}{b}+N_{i}\left(p_{i}-c+w_{i}-\eta p_{i}\right)\right. \\
=\phi_{j} \sum_{i=1}^{2} \frac{A^{2}\left[\left(p_{i}-c\right)+\left(w_{i}-\eta p_{i}\right)\right]^{2}}{2 b}+\frac{A^{2}\left[\left(p_{i}-c\right)+\left(w_{i}-\eta p_{i}\right)\right]^{2}}{2 b}
\end{gathered}
$$

As a result, the military enterprises and warehouses $i$ expected revenue under the transfer payment incentive mechanism is obtained:

$$
\begin{array}{ll}
\begin{array}{l}
\text { expected revenue under the transfer payment incentive } \\
\text { mechanism is obtained: }
\end{array} & \sum_{i=1}^{2} \pi_{s}^{N}\left(q_{i}\left(e_{i}\right)\right)=\sum_{i=1}^{2}\left\{\left[\frac{A^{2}\left[\left(\eta p_{i}-w_{i}\right)\left(p_{i}-c\right)-\left(\eta p_{i}-w_{i}\right)^{2}\right]}{b}+\right.\right. \\
\bar{\pi}_{s}\left(q_{1}\left(e_{1}\right), q_{2}\left(e_{2}\right)\right)=\sum_{i=1}^{2}\left[\pi_{s}^{J}\left(q_{i}\left(e_{i}\right)\right)-T\left(q_{i}\left(e_{i}\right)\right)\right] & \left.N_{i}\left[\left(p_{i}-c\right)+\left(w_{i}-\eta p_{i}\right)\right]\right\} \\
=\sum_{i=1}^{2}\left\{\frac{A^{2}\left(p_{i}-c\right)\left[\left(p_{i}-c\right)+\left(w_{i}-\eta p_{i}\right)\right]}{b}+N_{i}\left[\left[\left(p_{i}-c\right)+\left(w_{i}-\eta p_{i}\right)\right]\right\}\right. & \text { So, }
\end{array}
$$$$
\bar{\pi}_{s}\left(q_{1}\left(e_{1}\right), q_{2}\left(e_{2}\right)\right)=\sum_{i=1}^{2}\left[\pi_{s}^{J}\left(q_{i}\left(e_{i}\right)\right)-T\left(q_{i}\left(e_{i}\right)\right)\right]
$$

$$
\begin{gathered}
-\left(\phi_{1}+\phi_{2}+1\right) \sum_{i=1}^{2} \frac{A^{2}\left[\left(p_{i}-c\right)+\left(w_{i}-\eta p_{i}\right)\right]^{2}}{2 b} \\
\bar{\pi}_{r}\left(q_{i}\left(e_{i}\right)\right)=E\left[\pi_{i}^{J}\left(q_{i}\left(e_{i}\right)\right)+T\left(q_{i}\left(e_{i}\right)\right)\right] \\
=\left[\frac{A^{2}\left(p_{i}-c\right)\left(\eta p_{i}-w_{i}\right)}{b}+N_{i}\left(\eta p_{i}-w_{i}\right)-\frac{A^{2}\left(p_{i}-c\right)^{2}}{2 b}\right]+ \\
\phi_{j} \sum_{i=1}^{2} \frac{A^{2}\left[\left(p_{i}-c\right)+\left(w_{i}-\eta p_{i}\right)\right]^{2}}{2 b}+\frac{A^{2}\left[\left(p_{i}-c\right)+\left(w_{i}-\eta p_{i}\right)\right]^{2}}{2 b}
\end{gathered}
$$

$$
\begin{gathered}
\sum_{i=1}^{2}\left[\bar{\pi}_{s}\left(q_{i}\left(e_{i}\right)\right)-\pi_{s}^{N}\left(q_{i}\left(e_{i}\right)\right)\right] \\
=\left(1-\phi_{1}-\phi_{2}\right) \sum_{i=1}^{2} \frac{A^{2}\left[\left(p_{i}-c\right)+\left(w_{i}-\eta p_{i}\right)\right]^{2}}{2 b} \geq 0, \\
\sum_{i=1}^{2}\left[\pi_{r}\left(q_{i}\left(e_{i}\right)\right)-\pi_{r}^{N}\left(q_{i}\left(e_{i}\right)\right)\right]
\end{gathered}
$$

And because, 


$$
\begin{gathered}
=\left(\phi_{1}+\phi_{2}\right) \sum_{i=1}^{2} \frac{A^{2}\left[\left(p_{i}-c\right)+\left(w_{i}-\eta p_{i}\right)\right]^{2}}{2 b} \geq 0, \\
\sum_{i=1}^{2}\left[\bar{\pi}\left(q_{i}\left(e_{i}\right)\right)-\pi^{N}\left(q_{i}\left(e_{i}\right)\right)\right] \\
=\sum_{i=1}^{2} \frac{A^{2}\left[\left(p_{i}-c\right)+\left(w_{i}-\eta p_{i}\right)\right]^{2}}{2 b} \geq 0 .
\end{gathered}
$$

The above process shows that the linear transfer payment incentive mechanism can maximize the military supply chain revenue, as well as makes the military supply chain coordination and orderly operation, also can be applied to many warehouses. [14-15]

\section{Conclusion}

From the above paper model study, we can draw a more meaningful conclusion from different aspects:

(1) The warehouse effort level is no consensus under the decentralized decision and centralized decision making if there is no incentive mechanism, and the military supply chain can't also reach the coordination. Under the information symmetry, even if the military supply chain does not take any incentive measures, the rational warehouse will not hesitate to choose the optimal effort level to maximize their own revenue, namely the effort level in decentralized decision-making.

(2) According to the study analysis, we can get that the military enterprises and warehouses in the transfer payment incentive mechanism and revenue sharing mechanism gain more than under decentralized decision and no incentive mechanism, indicating that the transfer payment incentive mechanism can make the supply chain coordination. The military enterprises revenue not only achieves the maximization, but also the warehouse income achieves the optimization, and the military supply chain expected revenue achieves the maximization.

(3) From the view of the military supply chain whole revenue to maximize, design the incentive mechanism between the supply chain members, using the transfer payment means to change the warehouse expectation revenue goal function, allowing them to choose the optimal effort level the same as the military supply chain total expected revenue, each other establishes long-term cooperative partnership based on mutual trust and information sharing, give full play to their respective advantages, to achieve the improving the supply chain collaboration efficiency purpose, achieve the improving military logistic support ability goal in the end.

\section{References}

[1] LI Yan-bing, WANG Chuan-xu. Two stage supply chain optimization and coordination based on rate of profit to cost $[\mathrm{J}]$. Journal of Industrial Engineering and Engineering Management, 2018(1):100-106. (in Chinese)
[2] MA Xue-li, WANG Shu-yun, JIN Hui, BAI Qing-guo. Coordination and Optimization of Three-echelon Agricultural product Supply Chain Considering Freshness-keeping Effort and Quantity/Quality Elasticity [J]. Chinese Journal of Management Scienc, 2018, 26(2):175-185. (in Chinese)

[3] LIU Lang, SHI Wen-qiang, FENG Liang-qing. Supply Chain Coordination with Quantity Flexibility Contract under the Scene of Multi-factor Disturbance [J]. Chinese Journal of Management Science, 2016, 24(7):163-176. (in Chinese)

[4] WU Shuang-sheng, LIU Lang, SHI Wen-qiang, GONG Ling-jun. Emergency Quantity Flexibility Contract under Risk Aversion of a Supplier Considering Stochastic Price [J]. Soft Science, 2017, 31(11):128-133. (in Chinese)

[5] FENG Ying, ZHOU Ying. Supply Chain Decision and Coordination with Sales Efforts Effects on the Demand under VMCI [J]. Systems Engineering, 2017, 35(2):122-127. (in Chinese)

[6] ZHANG ting-long, FANG jin-jun. Competing and Coordination Strategies for Tourism Supply Chain under Revenue Sharing Contract [J]. Systems Engineering, 2017, 35(1):124-129. (in Chinese)

[7] LI Jian, YANG Yang. Supply Chain Coordination Model for Two-Stage Perishable Products of Permitted Emergency Ordering [J]. Statistics \& Decision, 2018(1):40-44. (in Chinese)

[8] DAI Jian-sheng. Revenue Sharing Contract for a Supply Chain with Demand Depending on Promotion and Pricing [J]. Chinese Journal of Management, 2018, 15(5):774-781. (in Chinese)

[9] SUN Jin-feng, ZOU Zong-bao. Decision-making of Product Price and Modularization Level and the Supply Chain Coordination [J]. Statistics \& Decision, 2018(3): 49-53. (in Chinese)

[10] CHEN Ge, DAN Bin, QIN Yan-hong. Supply Chain Coordination with Wholesale Price Contract and Fairness-concerned Agents [J]. Forecasting, 2017, 36(3):62-68. (in Chinese)

[11] CHEN Zhi-ming, CHEN zhi-xiang. Coordination the risk-averse OEM supply chain with random supply and demand [J]. Systems Engineering-Theory \& Practice, 2015, 35(5):1123-1132. (in Chinese)

[12] LIU Lang, SHI Yan. Coordination of Three-Stage Supply Chain with Unconventional Disruptions through Buy-Back Contract [J]. Journal of Systems \& Management, 2015, 24(2):296-303. (in Chinese)

[13] SHANG Wen-fang. Coordination and Contract Negotiation of Profit Sharing in Dual-channel Supply Chain [J]. Systems Engineering, 2015, 33(7):43-51. (in Chinese)

[14] ZENG Shun-qiu, LUO Jian-wen. Incentives Mechanism for Supply Chain Coordination with Trade Credit based on Quantity Discount [J]. Journal of Systems \& Management, 2015, 24(1):85-90. (in Chinese)

[15] WANG Xin-hui, WANG Xian-yu. The Coordination of Supply Chain with Bilateral Asymmetric Information by Considering Risk Aversion of Retailer [J]. Chinese Journal of Management Science, 2015, 23(3):97-107. (in Chinese) 